\title{
Nanoscale
}

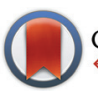

CrossMark \& click for updates

Cite this: Nanoscale, 2016, 8, 12658

\author{
Nalinikanth Kotagiri,,$^{a, b, c}$ Joshua Sakon, ${ }^{c, d}$ Haewook Han, ${ }^{\text {e,f }}$ Vladimir P. Zharov ${ }^{g}$ and \\ Jin-Woo Kimª,b,c
}

\section{Fluorescent ampicillin analogues as multifunctional disguising agents against opsonization $\dagger$}

\begin{abstract}
Cancer nanomedicines are opening new paradigms in cancer management and recent research points to how they can vastly improve imaging and therapy through multimodality and multifunctionality. However, challenges to achieving optimal efficacy are manifold starting from processing materials and evaluating their intended effectiveness on biological tissue, to developing new strategies aimed at improving transport of these materials through the biological milieu to the target tissue. Here, we report a fluorescent derivative of a beta-lactam antibiotic, ampicillin (termed iAmp) and its multifunctional physicobiochemical characteristics and potential as a biocompatible shielding agent and an effective dispersant. Carbon nanotubes (CNTs) were chosen to demonstrate the efficacy of iAmp. CNTs are known for their versatility and have been used extensively for cancer theranostics as photothermal and photoacoustic agents, but have limited solubility in water and biocompatibility. Traditional dispersants are associated with imaging artifacts and are not fully biocompatible. The chemical structure of iAmp is consistent with a deamination product of ampicillin. Although the four-membered lactam ring is intact, it does not retain the antibiotic properties. The iAmp is an effective dispersant and simultaneously serves as a fluorescent label for single-walled CNTs (SWNTs) with minimal photobleaching. The iAmp also enables bioconjugation of SWNTs to bio-ligands such as antibodies through functional carboxyl groups. Viability tests show that iAmp-coated SWNTs have minimal toxicity. Bio-stability tests under physiological conditions reveal that iAmp coating not only remains stable in a biologically relevant environment with high protein and salt concentrations, but also renders SWNTs transparent against nonspecific protein adsorption, also known as protein corona. Mammalian tissue culture studies with macrophages and opsonins validate that iAmp coating affords immunological resistance to SWNTs. Furthermore, iAmp coating offers protection to SWNTs against their nonspecific adsorption across disparate cell types, which has precluded a targeted strategy, and enables selective molecular targeting. The iAmp can therefore be used as an efficient dispersant, a photostable fluorescent agent, and a biocompatible disguising agent, alleviating CNTs' drawbacks and rendering them suitable for nanotheranostic and drug delivery applications.
\end{abstract}

Received 7th December 2015 Accepted 12th February 2016

DOI: $10.1039 / \mathrm{c} 5 \mathrm{nr} 08686 \mathrm{~h}$

www.rsc.org/nanoscale

\section{Introduction}

University of Arkansas, Fayetteville, AR, USA. E-mail: jwkim@uark.edu

${ }^{b}$ Department of Biological \& Agricultural Engineering, University of Arkansas, Fayetteville, AR, USA

${ }^{c}$ Cell \& Molecular Biology Graduate Program, University of Arkansas, Fayetteville, $A R, U S A$

${ }^{d}$ Department of Chemistry \& Biochemistry, University of Arkansas, Fayetteville, AR, USA

${ }^{e}$ Department of Electrical Engineering, Pohang University of Science \& Technology, Pohang, Republic of Korea

${ }^{f_{S c h o o l}}$ of Interdisciplinary Bioscience \& Bioengineering, Pohang University of Science \& Technology, Pohang, Republic of Korea

${ }^{g}$ Arkansas Nanomedicine Center, University of Arkansas for Medical Sciences, Little Rock, AR, USA

$\dagger$ Electronic supplementary information (ESI) available: Details of quantum yield estimation and physicobiochemical analyses to assess the mechanism of iAmp synthesis and its chemical structure as well as the cytotoxicity and bio-stability of iAmp and iAmp-SWNT hybrids. See DOI: 10.1039/c5nr08686h

¥Current address: Department of Radiology, Washington University School of Medicine, St. Louis, MO, USA.
Recent advances in cancer nanomedicine and theranostics have been greatly facilitated by complementary advances in materials science and related techniques. Engineered materials, through their novel properties, have been shown to enhance cancer detection and treatment outcomes in a multitude of preclinical studies and some have even progressed to clinical trials. ${ }^{1}$ Nanoparticulates and related nanosystems incorporate a multitude of biological and synthetic moieties to afford superior theranostic capabilities. The multimodality approach and the modularity of these nanosystems can potentially expand the magnitude and scope of cancer theranostics to include diverse cancer types and diseases through simple modulations in synthesis and formulation techniques. However, translating the advances in material formulation towards effective cancer nanomedicines has faced significant 
obstacles due to a limited understanding of the interaction of these engineered materials with the biological environment. While there have been a plethora of studies showing the effectiveness of novel engineered materials, as a therapeutic or imaging agent or both, ${ }^{2}$ determining their dispersity, biocompatibility, bio-stability is absolutely vital and inextricably linked to their effective transport in vivo during translational efforts. Particularly, the role of the immune system is of paramount importance since materials, which are inherently foreign to the biological environment, are primed for rapid elimination from the body. Phagocytosis by monocytes and macrophages dictates the fate of potential theranostic targeting platforms in circulation. ${ }^{3}$ These phagocytes are part of the innate immune system along with opsonins, i.e., plasma proteins such as complement factors and immunoglobulins, which attach nonspecifically to foreign objects and tag them for recognition by macrophages for phagocytosis and sequestration to the liver and spleen. ${ }^{4}$ In addition, nonspecific adsorption of many other proteins in biological fluids, known as protein corona, critically affects the interaction of particles with living systems, plays a decisive role in their biodistribution and biokinetics, and inevitably controls their in vivo fate. $^{3,5}$ Particles that are inert or possess favorable surface properties will not only escape opsonins and macrophages but also alleviate the protein corona formation and have a longer circulation half-life and enhanced biodistribution. ${ }^{3,6,7}$

Here, we introduce a fluorescent derivative of ampicillin (termed iAmp), a beta-lactam antibiotic, and its physicobiochemical characterization. We demonstrate that the iAmp plays a triple role as a highly effective dispersant, a photostable fluorescent labeling agent, and a biocompatible disguising (stealth) agent against innate immune responses as well as protein corona, to alleviate the drawbacks of nanomaterials and enables us to realize their full potential for biological and biomedical applications, particularly nanotheranostics and drug delivery.

As a proof-of-concept, we selected carbon nanotubes (CNTs), widely known for their superlative properties and a challenging material to process for biomedical applications. ${ }^{8}$ CNTs, particularly singled-walled CNTs (SWNTs), and their hybrids have shown great potential for cancer theranostics such as photothermal (PT) therapy, photoacoustic (PA) imaging, drug delivery, as well as reducing the load of bacteremia among many explorative studies. ${ }^{9-24}$ The challenges ahead partly stem from unresolved issues such as their poor biocompatibility, low solubility due to their hydrophobicity, and dispersibility in biologically relevant physiological solvents. $^{25-27}$ One of the most common strategies to render CNTs biocompatible and dispersible in aqueous solutions is to use shielding agents such as surfactants, polymers, polysaccharides, proteins, and DNA. ${ }^{3,18,24,28-30}$ Among the shielding agents, the most commonly used are surfactants. However, surfactant dispersed CNTs are often difficult to handle since significant flocculation can occur in inadequately optimized preparations, ${ }^{31}$ thus compromising imaging and downstream processes.
Besides improving the solubility and biocompatibility of CNTs, there are other considerations such as interactions with body fluids (i.e., human blood, plasma, and serum) and circulation half-life that will be impacted directly by the coating material. CNTs are known to be recognized by the body's immune system and are primed for quick elimination. ${ }^{32}$ To realize the potential of CNTs for pharmaceutical and medical theranostic applications, novel coatings that can render CNTs transparent to the immune system are required. Traditionally, polyethylene glycol (PEG) is used to help CNTs evade the protein corona as well as the mononuclear phagocyte system (MPS). As noticed in many synthetic materials, there are risks of an adverse reaction against PEG. Recently, PEG-SWNT hybrids are not only reported to elicit concentration and surface density dependent cytotoxicity caused by oxidative stress, ${ }^{33}$ but phospholipid-PEG moieties are also shown to be responsible for complement activation by CNTs, triggering the lectin pathway. ${ }^{34}$ Hence, there is a need for alternative shielding strategies that provide not only biocompatibility and solubility in biologically relevant solutions but also a "stealth" character to CNTs.

Fluorescent ampicillin-like molecules have been synthesized in the past. ${ }^{35}$ However, complex reaction conditions were required. Moreover, the chemical structures of these derivatives were not fully characterized. Our reported technique is much simpler and cost effective. Several groups have demonstrated the adsorption of various antibiotics, such as sulfonamides, quinolones, anthracyclines and beta-lactams, on CNTs through $\pi-\pi$ interaction. ${ }^{36-39}$ Antibiotic-coated CNTs were primarily intended for anti-bacterial and anti-cancer treatment, where CNT served as a transporter of the active agent. In this study, we have used an inactivated antibiotic, i.e., iAmp, which has properties distinct from those of the parent compound, primarily for the structural and functional enhancement of CNTs. Specifically, we used the iAmp to disperse and render SWNTs biocompatible and fluorescent. We also demonstrated its utility particularly for nanotheranostics by showing that the iAmp is able to disguise SWNTs from opsonins and prevent phagocytosis by macrophages. The work here thus shows iAmp as a highly biocompatible and versatile shielding agent, offering much promise for the realization of nanoparticle-based nanotheranostics and drug delivery.

\section{Experimental section}

\section{Materials}

All water used was purified and deionized with an EASY pure RF system (Barnstead, Dubuque, IA; $18.2 \mathrm{M} \Omega \mathrm{cm}$ ). SWNTs were purchased from Carbon Nanotechnologies Inc. (CNI) (Houston, TX; CNI Grade/Lot number: P0274). Ampicillin, ammonia assay kit, complement factor $\mathrm{C} 3 \mathrm{~b}$, anti-rabbit immunoglobulin G (IgG), anti-rabbit IgG conjugated with fluorescein isothiocyanate (IgG-FITC), and other reagents and solvents, unless specified, were purchased from Sigma-Aldrich (Milwaukee, WI), CellTiter 96® AQueous One Solution Cell 
Proliferation Assay from Promega (Madison, WI), 1-ethyl-3-[3dimethylaminopropyl]carbodiimide hydrochloride (EDC) and $N$-hydroxysulfosuccinimide (Sulfo-NHS) from Pierce Biotechnology (Rockford, IL), and Escherichia coli K-12, human macrophage, T98G human glioblastoma cell line (CRL-1690 ${ }^{\mathrm{TM}}$ ), and their media from American Type Culture Collection (ATCC) (Manassas, VA).

\section{Synthesis of iAmp}

The iAmp was synthesized by heating a fresh solution of ampicillin consisting of $5 \mathrm{mg}$ of ampicillin and $1 \mathrm{ml}$ of water, unless specified, in a thermal cycler (Bio-Rad ${ }^{\circledR} \mathrm{S}-1000^{\mathrm{TM}}$ Thermal Cycler, Bio-Rad Laboratories, Inc., Hercules, CA) for $2 \mathrm{~h}$ at $99^{\circ} \mathrm{C}$. To prevent any evaporation, the lid was set to a temperature of $100{ }^{\circ} \mathrm{C}$. The iAmp solutions were desiccated using a RapidVap Vacuum Dry Evaporation System (Labconco, Kansas City, MO).

\section{Dispersion of SWNTs with iAmp}

The reaction mixture consisted of $1 \mathrm{mg}$ of pristine SWNTs and $1 \mathrm{ml}$ of water to which $50 \mu \mathrm{l}$ of iAmp was added. Likewise, another reaction mixture consisting of $1 \mathrm{mg} \mathrm{ml}^{-1}$ of dispersed and shortened SWNTs (dsSWNTs) with the same concentration of iAmp was also prepared. The dsSWNTs were synthesized as reported previously. ${ }^{18,24}$ The reaction mixtures were subjected to sonication in a bath sonicator (VWR, West Chester, PA) for $20 \mathrm{~min}$. After sonication, the iAmp-SWNT adducts were purified from unbound iAmp using a Harvard Apparatus Ultra-Fast Dialyzer (Holliston, MA) with a cellulose acetate membrane [2 kDa molecular weight cut-off (MWCO)] in water for $24 \mathrm{~h}$ at $25^{\circ} \mathrm{C}$.

\section{Antibiotic sensitivity test}

Two Luria-Bertani (LB) agar ( $\mathrm{pH} \mathrm{7;} \mathrm{ATCC)} \mathrm{plates,} \mathrm{one} \mathrm{with}$ $50 \mu \mathrm{g} \mathrm{ml}{ }^{-1}$ of ampicillin and the other with $50 \mu \mathrm{g} \mathrm{m} \mathrm{m}^{-1}$ of iAmp, were streaked with the E. coli K-12 strain. The plates were then incubated at $37^{\circ} \mathrm{C}$ for $14 \mathrm{~h}$.

\section{Cytotoxicity and bio-stability tests}

The cytotoxicity of iAmp and iAmp-coated SWNTs was assessed by monitoring the cell viability and proliferation for 10 days using CellTiter 96® AQueous One Solution Cell Proliferation Assay with a human glioblastoma cell line according to the specifications by Promega and ATCC. All experiments were performed in compliance with guidelines set by the Institutional Biosafety Committee (IBC) at the University of Arkansas and approved by the IBC. Briefly, the cell line was cultured in Eagle's Minimum Essential Medium (EMEM) supplemented with $10 \%$ fetal bovine serum at $37{ }^{\circ} \mathrm{C}$ and $5 \% \mathrm{CO}_{2}$. The incubations of iAmp and iAmp-SWNTs with the cell line were carried out in 12-well plates, containing the cells seeded for $24 \mathrm{~h}$ before applying iAmp or iAmp-SWNTs. Control cells without iAmp and iAmp-SWNTs or cells treated with iAmp or iAmp-SWNTs at various concentrations $\left(10-100 \mu \mathrm{g} \mathrm{ml}^{-1}\right.$ and 100-500 $\mu \mathrm{g} \mathrm{ml} \mathrm{ml}^{-1}$ as final concentrations, respectively) were incubated for 10 days at $37^{\circ} \mathrm{C}$ and $5 \% \mathrm{CO}_{2}$. After incubation, the cell proliferation assay was performed for each sample and the cell viability profile for each sample was assessed by colorimetric absorbance at $490 \mathrm{~nm}$ using a DU-800 ultraviolet/ visible/NIR (UV/vis/NIR) spectrophotometer (Beckman Coulter Inc., Fullerton, CA). The extended incubation time of 10 days was chosen to allow sufficient time for the influence, if any, of iAmp or iAmp-SWNTs on cell viability.

The bio-stability of iAmp-SWNTs was evaluated by testing whether iAmp-SWNTs remained stable in the biologically relevant environment after incubating them in EMEM with 10\% FBS at $37{ }^{\circ} \mathrm{C}$ and $5 \% \mathrm{CO}_{2}$ for 10 days. Controls, including SWNTs only in water and iAmp-SWNTs in 0.1 M PBS buffer ( $\mathrm{pH}$ 7.4), were also incubated for 10 days at $37^{\circ} \mathrm{C}$ and $5 \% \mathrm{CO}_{2}$. After incubation, the samples were purified using the Harvard Apparatus Ultra-Fast Dialyzer with a $50 \mathrm{kDa}$ MWCO cellulose acetate membrane in 0.1 M PBS buffer ( $\mathrm{pH} 7.4)$ for $24 \mathrm{~h}$ at $25{ }^{\circ} \mathrm{C}$. The bio-stability of the iAmp coating was determined by assessing the fluorescence intensity of iAmp-SWNTs in each sample using fluorospectrometry and their dispersity using Atomic Force Microscopy (AFM) as detailed below. Again, the 10-day extended incubation time was chosen to allow sufficient time for the influence, if any, of the physiological conditions containing high protein and salt concentrations (e.g., that in blood) on the stability of iAmp coating.

\section{Ab bioconjugation to iAmp-SWNTs}

Carbodiimide chemistry was implemented according to the manufacturer's instructions (Pierce Biotechnology, Rockford, IL) in order to conjugate the carboxyl group at C-22 of the iAmp on the SWNT hybrid to the amine group on IgG and IgG-FITC. After the conjugation reaction, excess EDC, SulfoNHS, and free Ab were removed using the Harvard Apparatus Ultra-Fast Dialyzer with a $300 \mathrm{kDa}$ MWCO cellulose acetate membrane in 0.1 M PBS buffer ( $\mathrm{pH} 7.4)$ for $24 \mathrm{~h}$ at $4{ }^{\circ} \mathrm{C}$.

\section{Interaction of macrophages and opsonins with iAmp-SWNT hybrids}

Human macrophages were purchased from ATCC. All experiments were performed in compliance with the IBC guidelines and approved by the IBC. Macrophages were cultured according to the vendor's specifications and kept in their original growth medium (Iscove's modified Dulbecco's medium) for the entirety of the experiments. The iAmp-SWNTs and FITC tagged iAmp-SWNT-IgG complexes along with the dsSWNT control were preincubated with opsonins, IgG-FITC and/or $\mathrm{C} 3 \mathrm{~b}$, at $37^{\circ} \mathrm{C}$ for $2 \mathrm{~h}$. After incubation, they were added to the medium containing macrophages and mixed for $2 \mathrm{~h}$ at $37^{\circ} \mathrm{C}$. The samples were then washed (centrifugation at $2200 \mathrm{~g}$ for 5 min followed by medium exchange) three times to remove any unbound particles.

\section{Physicochemical characterization}

The absorption spectra of samples were obtained using a DU-800 UV/Vis/NIR spectrophotometer (Beckman Coulter Inc., Fullerton, CA) and the fluorescence spectra using a NanoDrop 
3300 Fluorospectrometer (Thermo Fisher Scientific, Inc., Wilmington, DE).

Atomic force microscopy (AFM) imaging was carried out with a Veeco Multimode Scanning Probe Microscope with a Nanoscope IIIa Controller (Veeco Instruments, Woodbury, NY). For AFM sample preparation, $25 \mu$ l of each sample solution was mixed with $25 \mu \mathrm{l}$ of DI water and $5 \mu \mathrm{l}$ of the mixture was dispensed on a mica substrate (Novascan, Ames, IA). All samples were scanned in the tapping mode in air with a NanoWorld Pointprobe ${ }^{\circledR}$ NCSTR AFM probe (NanoWorld AG, Neuchâtel, Switzerland), which is designed for soft tapping mode imaging and enables stable and accurate measurements with a reduced tip-sample interaction, in order to obtain highresolution AFM images with minimal sample damage. The sample scan rate was $1.0 \mathrm{~Hz}$ with an aspect ratio of $1: 1$. The force constant of the tip for scanning was $7.4 \mathrm{~N} \mathrm{~m}^{-1}$. The free resonance frequency of the cantilever was automatically tuned by Nanoscope Software (version v5.31r1; Veeco Instruments).

Phase-contrast and transmittance microscopy were performed using a light microscope (Axioskop 2 Plus, Carl Zeiss, Inc., Germany) equipped with a 12-bit Color MicroImager II Cooled digital camera (QImaging, Burnaby, Canada) with a resolution of 1.3 million pixels. The $100 \times$ or $63 \times$ oil immersion objectives (Carl Zeiss) were used to visualize and acquire the images and their sequences with a digital image and video recording software, StreamPix (Norpix, Inc., Montreal, Canada). The light microscopy system was additionally equipped with two filter sets consisting of band-pass filters covering 450 to $490 \mathrm{~nm}$ (FITC, Carl Zeiss) and 350 to $370 \mathrm{~nm}$ (DAPI, Carl Zeiss) for exciters and absorbance filters covering wavelengths of $515 \mathrm{~nm}$ (FITC, Carl Zeiss) and $400 \mathrm{~nm}$ (DAPI, Carl Zeiss).

\section{Results and discussion}

\section{Synthesis and physicobiochemical characterization of iAmp and its SWNT hybrid}

Ampicillin belongs to the penicillin group of beta-lactam antibiotics, differing from penicillin $\mathrm{G}$ by a single amine group. The heating of a clear and colorless ampicillin solution to $99{ }^{\circ} \mathrm{C}$ resulted in an amber colored iAmp solution (Fig. 1a, left). The iAmp was highly water-soluble and the compound did not show any signs of precipitation in water over long time periods, i.e., $\geq 6$ months. Desiccation left a yellow colored powder unlike its parent compound, which is white (Fig. 1a, right). The absolute yield of iAmp as the weight in grams was very high ( $>98 \%)$ after desiccation, based on mass measurements. The solution emitted intense blue light on irradiation with UV light (Fig. 1a, middle). The fluorescence quantum yield $(\Phi)$ of iAmp was estimated to be 0.11 (see the ESI $\dagger$ ). The iAmp had a unique absorption and emission maxima at $340 \mathrm{~nm}$ and $435 \mathrm{~nm}$, respectively (Fig. 1b), not exhibited by ampicillin. The iAmp dissolved in water and kept in a clear container under ambient light conditions for $\geq 6$ months had a less than $5 \%$ loss in its absorbance and fluorescence.
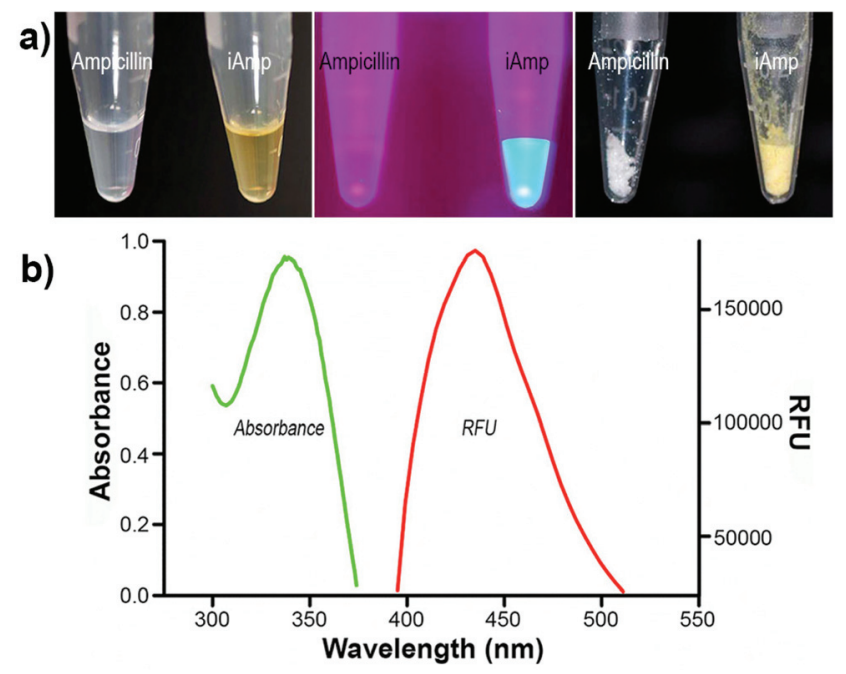

Fig. 1 (a) Ampicillin and iAmp solutions in the ambient (left) and UV (center) lights and their desiccated samples (right). (b) UV/Vis absorption (green line; left axis) and fluorescence (red line; right axis) spectra of iAmp solution. RFU indicates relative fluorescence units.

Pure CNTs are highly aggregated, long, and bundled structures that are held together by weak van der Waals forces. The iAmp-coated SWNT solution, obtained on sonicating an admixture of pristine SWNTs with iAmp, acquired a uniform dark coloration, suggesting that there was a profound increase in the solubility of SWNTs (Fig. S1 in the ESI $\dagger$ ). The iAmpcoated SWNTs remained photostable and dispersed in water. The UV/vis absorption spectrum displayed the characteristic Van Hove peaks of SWNTs, suggesting the separation of bundles and complete dispersion in an aqueous solvent as individual tubes (Fig. 2a, red line). This phenomenon was unique to iAmp and was observed neither for ampicillin (Fig. 2a, green line) nor for other beta lactam antibiotics such as amoxycillin and penicillin G (Fig. S1 $\dagger$ ). AFM was used to further analyze the high degree of dispersion after purification of iAmp-SWNT conjugates from unbound iAmp. AFM scans of pristine SWNTs coated with iAmp showed uniformly dispersed long SWNTs on the mica surface (Fig. 2b, left). AFM section analysis revealed the diameter to be $1.2 \mathrm{~nm}$ on an average (Fig. 2c, top). Given the diameter of individual SWNTs is 0.8-1.2 nm, this result confirms that SWNTs were disbanded completely. In contrast, dispersed and shortened SWNTs (dsSWNTs) in water (i.e., neither surfactants nor iAmp), prepared through sonication in an acid mixture $\left(\mathrm{H}_{2} \mathrm{SO}_{4}+\right.$ $\left.\mathrm{HNO}_{3}\right),{ }^{18,24}$ did not display the Van Hove peaks; thus, were not completely unbundled. Conventionally, surfactants such as triton-X, sodium dodecyl sulfate (SDS), and sodium dodecylbenzenesulfonate (NaDBS) are used to fully disperse SWNTs after such chemical oxidation. ${ }^{29,30,40}$ However, the surfactantcoated SWNTs, besides proving cumbersome for high resolution scanning probe and electron microscopy, can denature lipids and proteins, thus precluding them from biomedical studies. Also, surfactants do not confer any additional traits, which could otherwise potentially expand the range of 
a)

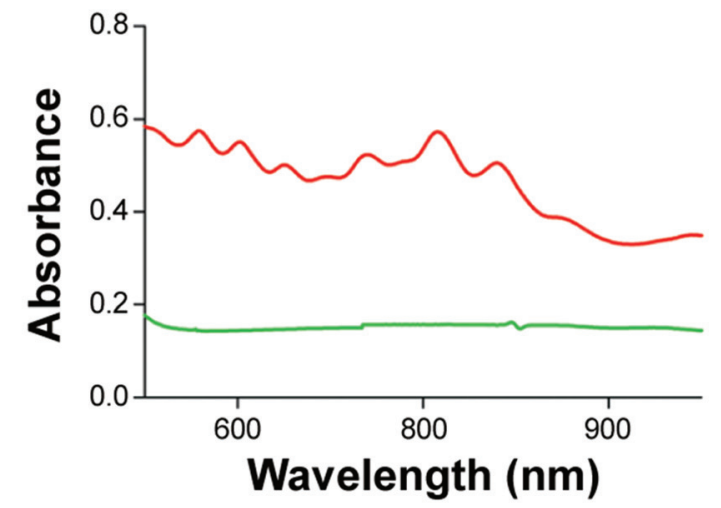

b)
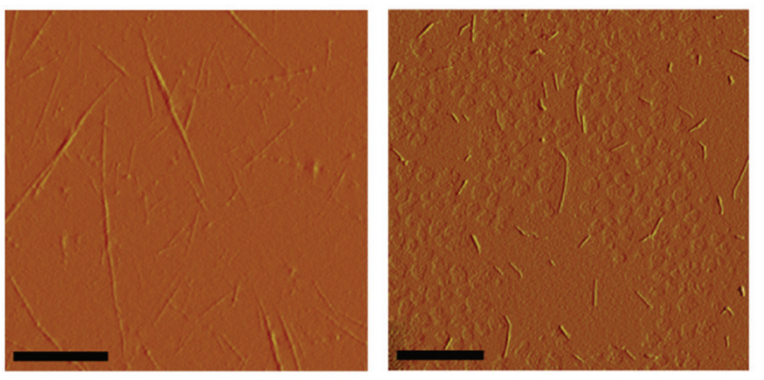

c)
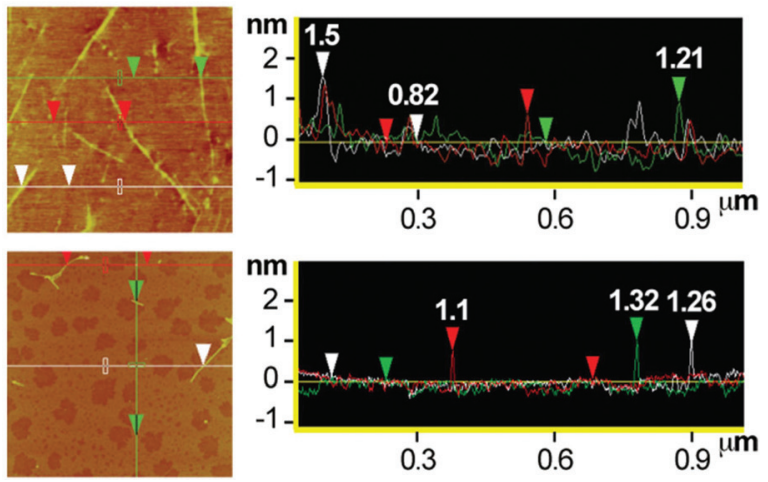

Fig. 2 (a) Vis/NIR absorption spectra of iAmp-SWNTs (red line) and ampicillin-SWNTs (green line). (b) Phase AFM images of pristine SWNTs (left) and dsSWNTs (right) with iAmp. Scale bars represent $400 \mathrm{~nm}$ (left) and $200 \mathrm{~nm}$ (right). (c) AFM height images (left) and section analysis plots (right) showing the vertical heights, representatives of the diameters, of iAmp-dispersed pristine SWNTs (top) and dsSWNTs (bottom). The average diameter is $\sim 1.2 \mathrm{~nm}$.

applications. After treating dsSWNTs in water with iAmp, AFM scans yielded a similar result as iAmp-treated pristine SWNTs (Fig. 2b, right), displaying uniformly dispersed SWNTs with a diameter of $1.2 \mathrm{~nm}$ on an average (Fig. 2c, bottom). For the rest of the study, the iAmp-coated dsSWNT hybrids were used only because of the relative size uniformity of dsSWNTs as reported previously. ${ }^{30}$

To understand the binding mechanism of iAmp to SWNTs, its structure was elucidated. ${ }^{1} \mathrm{H}$ nuclear magnetic resonance (NMR) spectroscopy was performed on iAmp dissolved in $\mathrm{D}_{2} \mathrm{O}$. As shown in Fig. S2 in the ESI, $\dagger$ the chemical shifts are labeled according to the corresponding hydrogen atoms. A comparison of the ${ }^{1} \mathrm{H}$ NMR spectrum of ampicillin (Fig. S2a in the ESI $\dagger$ ) and that of iAmp (Fig. S2b in the ESI $\dagger$ ) highlighted that $\mathrm{H}-10$ was absent in iAmp, while all the other chemical shifts were invariant. The structure of iAmp must resemble ampicillin except for $\mathrm{H}-10$. Isoelectric focusing demonstrated that the net charge of iAmp is negative (Fig. S3 in the ESI $\dagger$ ), perhaps due to the loss of an amine group at C-10. Yet, Fourier transform infrared (FTIR) spectroscopy performed on dried samples of iAmp and ampicillin resembled each other (Fig. S4 in the ESI $\dagger$ ). The high-performance liquid chromatography (HPLC) chromatograms of iAmp showed distinct absorption peaks at $340 \mathrm{~nm}$ (Fig. S5 and S6 in the ESI†). Subsequent liquid chromatography-mass spectrometry (LC-MS) yielded the molecular weight (MW) of iAmp to be 348.8 Da (Fig. S7, top in the ESI†) and the MW of ampicillin to be $349.7 \mathrm{Da}$ (theoretical mass is $349.4 \mathrm{Da}$ ) (Fig. S7, bottom in the ESI†). One less nitrogen atom in iAmp was assumed based upon the "nitrogen rule". ${ }^{41}$

The $\mathrm{pH}$ of the ampicillin solution was measured as 7.7 and that after the formation of iAmp upon heating was 8.75. This increase in $\mathrm{pH}$ could partly be due to the consequence of deamination. An ammonium assay was performed to quantitate ammonia in solution (see the ESI $\dagger$ ). Ammonia is measured as a function of oxidation of nicotinamide adenine dinucleotide phosphate (NADPH). A decrease in the absorbance of the sample at $340 \mathrm{~nm}$ is an indicator of ammonia in solution. Upon modifying $5 \mathrm{mg} \mathrm{ml}^{-1}$ of ampicillin to iAmp, $0.27 \mathrm{mg} \mathrm{ml}^{-1}$ of ammonia was produced. The chemical reaction can be written as:

$$
\mathrm{C}_{16} \mathrm{H}_{19} \mathrm{~N}_{3} \mathrm{O}_{4} \mathrm{~S}+\mathrm{H}_{2} \mathrm{O} \stackrel{\Delta}{\longrightarrow} \mathrm{C}_{16} \mathrm{H}_{17} \mathrm{~N}_{2} \mathrm{O}_{5} \mathrm{~S}+\mathrm{NH}_{3}+\mathrm{H}^{+}+\mathrm{e}^{-}
$$

Evidence is consistent with the occurrence of oxidative deamination at the $\mathrm{C}-10$ position of ampicillin, resulting in fluorescent iAmp (Fig. 3a). The extended conjugate system of iAmp not only imparts the unique blue fluorescence but also facilitates $\pi-\pi$ interaction with SWNTs (Fig. 3b). As reported earlier, $\pi-\pi$ interaction of small molecules with SWNTs is a stable interaction, preventing desorption from the nanotube surface in aqueous solutions over prolonged durations. ${ }^{42}$ Noncovalent functionalization of small molecules such as pyrene also resulted only in a partial loss of fluorescence upon binding to SWNTs, due to charge transfer mechanisms. ${ }^{43}$ The imaging and tracking of noncovalently bound pyrene-SWNT conjugates, in situ, using fluorescence microscopy was still highly feasible, notwithstanding the moderate degree of fluorescence quenching. ${ }^{30,40}$ Similarly, epi-fluorescence microscopy was used to evaluate the in situ photostability of iAmp-SWNTs. The iAmp-SWNTs were visualized at $100 \times$ magnification using the DAPI filter. Bright rod-shaped fluorescent particles were observed with an exposure time of $250 \mathrm{~ms}$ (Fig. 3c) with a minimal fluorescence quenching. The long fluorescence halflife could be due to minimal spectral overlap in the emission spectra of iAmp and absorption spectra of SWNTs. The advantage of using iAmp is that it can disperse SWNTs, in addition to serving as a fluorescent tag.

Of note is the fact that the iAmp exhibited a marked decrease in its fluorescence intensity under acidic conditions. A $\sim 50 \%$ decrease in the intensity at $\mathrm{pH}=4.4$ was registered 

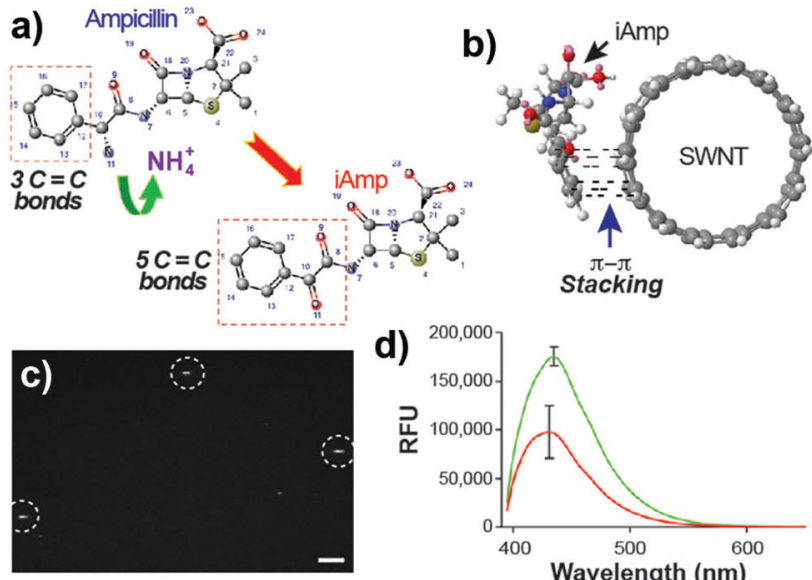

e)
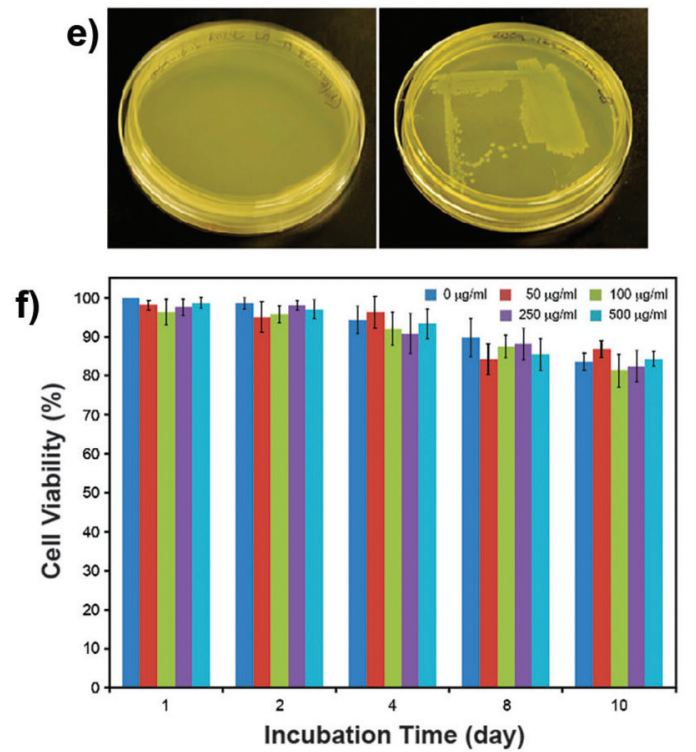

Fig. 3 (a) Schematic of ampicillin transforming into iAmp. (b) Schematic of iAmp $\pi$-stacking on SWNT. Note that the schematics do not reflect the actual object sizes. (c) Epi-fluorescence image of iAmp-SWNTs in PBS. Scale bar represents $500 \mathrm{~nm}$. (d) Fluorescence spectra of iAmp in an acidic solution of $\mathrm{pH} 4.4$ (red line) and a neutral solution of $\mathrm{pH} 7.2$ (green line). (e) LB agar plates with ampicillin and E. coli K12 (left) and iAmp and E. coli K12 (right) after $14 \mathrm{~h}$ incubation at $37^{\circ} \mathrm{C}$. (f) Cytotoxicity studies of iAmp-SWNTs. Note that all experiments were performed 3 times in triplets and the average has been shown as a cell-viability percentage in comparison with the iAmp-SWNT untreated control samples at time 0 . The controls at time 0 were considered as $100 \%$ viable. The SWNT, ampicillin, and iAmp models were prepared using Chem3D and the ChemDraw Pro programs (CambridgeSoft, Cambridge, MA).

(Fig. 3d). The fluorescence intensity returns to normal on titrating the $\mathrm{pH}$ back to 7.2. A similar behavior was observed with the iAmp-SWNT complex as well. One can envisage the use of these complexes as pH sensitive sensors.

\section{Antibiotic activity, cytotoxicity and bio-stability of iAmp and its SWNT hybrid}

Since iAmp has an intact beta-lactam ring, we expected it to retain the antibiotic activity of ampicillin. Bacterial suscepti- bility studies using the E. coli K-12 strain, which is sensitive to ampicillin, were conducted. Two sets of LB agar plates, one containing iAmp and the other ampicillin, were streaked with the K-12 strain and grown for $14 \mathrm{~h}$ at $37^{\circ} \mathrm{C}$. The plates with ampicillin showed no growth (Fig. 3e, left) compared to the normal growth of colonies on the iAmp plates (Fig. 3e, right), suggesting that iAmp does not possess the antibiotic properties of its parent compound. It is possible that the uptake of iAmp by bacteria is reduced due to its structural modification. Specifically, the loss of the amino group has the potential to greatly attenuate its uptake in Gram-negative bacteria such as E. coli.

Cell viability and proliferation assays revealed no apparent adverse toxicity effects on live cells after their exposure to various concentrations of iAmp and iAmp-SWNTs for 10 days (Fig. $3 \mathrm{f}$ and $\mathrm{S} 8$ in the ESI $\dagger$ ), suggesting that the iAmp coating improves the biocompatibility of CNTs with minimal cytotoxicity. No non-specific association between the mammalian cells and iAmp-SWNT adducts, after the 10-day incubation period, was observed, implying that the iAmp coating offers protection to SWNTs against their nonspecific adsorption across disparate cell types, therefore enabling selective molecular targeting by functionalizing the adducts with biomarker specific ligands.

The bio-stability of iAmp-SWNTs was further tested under physiological conditions containing high salt and protein concentrations found in the blood, by exposing them to PBS and EMEM with $10 \% \mathrm{FBS}$ at $37^{\circ} \mathrm{C}$ and $5 \% \mathrm{CO}_{2}$ for 10 days, respectively. Fluorescence measurements revealed that the fluorescence intensity of iAmp on SWNTs remained stable even after 10 days of incubation (Fig. S9a-c in the ESI $\dagger$ ). Also, AFM scans yielded a similar result as the control iAmp-SWNTs (Fig. 2b), displaying uniformly dispersed SWNTs without noticeable aggregations or association of proteins (Fig. S9d in the ESI $\dagger$ ). This indicates that the iAmp coating, through $\pi-\pi$ interaction with SWNTs, remains stable in such a high protein and salt environment, which typically displaces surface moieties rapidly from nanoparticles if the associations are weak. Also, this suggests that the iAmp coating could allow SWNTs to possess favorable surface properties, alleviating protein corona formation and enhancing circulation persistence and biodistribution.

\section{Functionalization of iAmp-SWNT for bio-specificity}

The carboxyl group at C-22 (Fig. 3a) can be used for bioconjugation reactions. We used carbodiimide chemistry to link the carboxyl group of iAmp-SWNTs to the amine groups of $\mathrm{Ab}$ using IgG (Fig. 4a). AFM images revealed that the successful covalent conjugation of $\mathrm{Ab}$ resulted in non-uniform 'beaded' surface features on SWNTs (Fig. 4b). Section analysis confirmed the association through a significant increase in the diameter $(7.1 \mathrm{~nm} \pm 0.69 \mathrm{~nm})$ of the iAmp-SWNT-IgG construct (Fig. 4c). AFM section analysis of IgG alone showed its average height of $3.5 \mathrm{~nm}( \pm 0.93 \mathrm{~nm})$ (Fig. S10 in the ESI $\dagger)$. The Y-shaped IgG is a flexible molecule with its typical dimensions of $14.5 \mathrm{~nm} \times 8.5 \mathrm{~nm} \times 4.0 \mathrm{~nm} .{ }^{44}$ The discrepancy in the 
a)
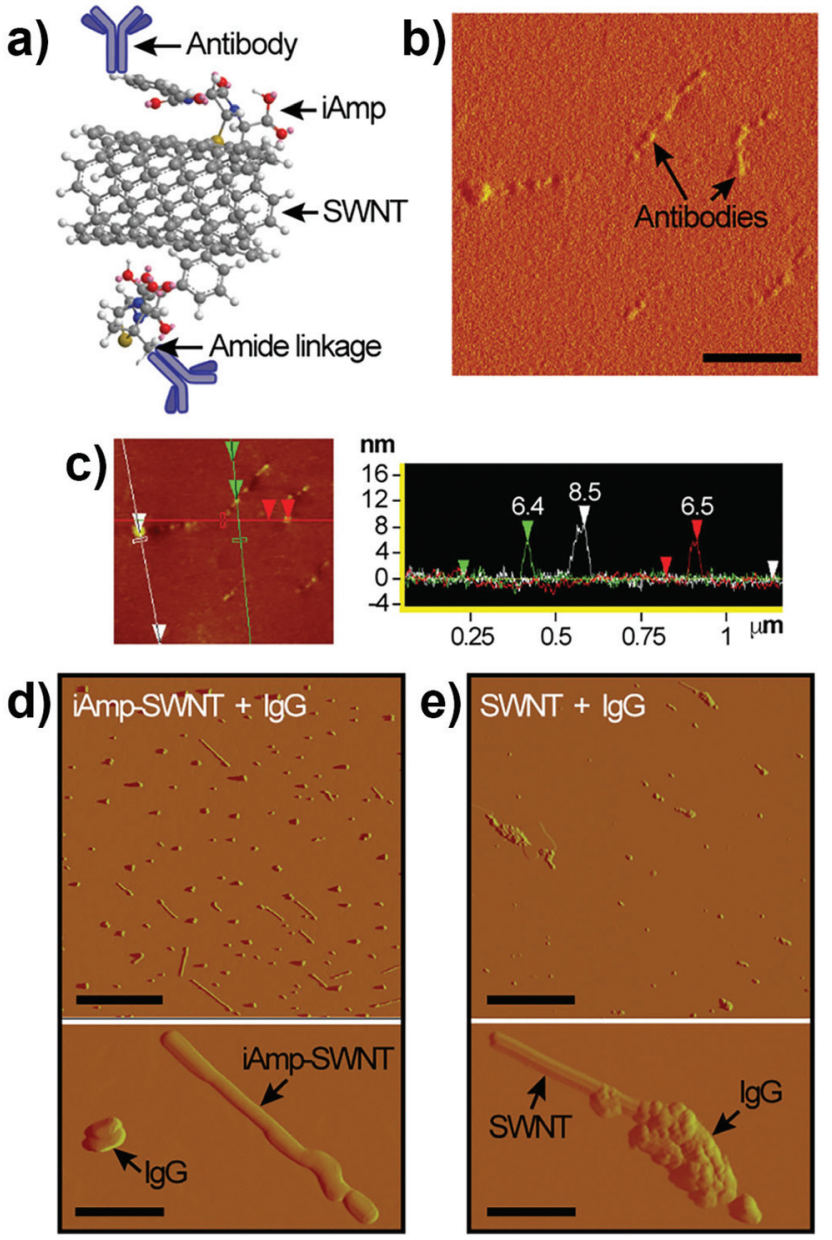

Fig. 4 (a) Schematic of the covalent carbodiimide-based coupling of $A b$ to iAmp-SWNT. Note that the schematic does not reflect the actual object sizes. (b) Phase AFM image of iAmp-SWNTs after Ab conjugation (iAmp-SWNTs-lgG). Notice the uneven 'bumpy' surfaces of SWNTs in contrast to iAmp-SWNTs in Fig. 2b, right. Scale bar represents $200 \mathrm{~nm}$. (c) AFM height image (left) and section analysis plot (right) showing the diameter of three different iAmp-SWNT-IgG constructs in the field. Notice the marked increase in the diameter compared to that of iAmpSWNTs in Fig. 2c. The average diameter is $\sim 7.1 \mathrm{~nm}$. (d) Phase AFM images of iAmp-SWNT admixed with IgG to observe their non-specific interaction. Notice iAmp-SWNT adducts can be identified as distinctly separate from the unbound IgG moieties (top). Scale bar represents $500 \mathrm{~nm}$. Magnified phase AFM image clearly demonstrates the absence of nonspecific adsorption of IgG on the surface of iAmp-SWNT (bottom). Scale bar represents $100 \mathrm{~nm}$. (e) Phase AFM images of dsSWNT admixed with IgG. Notice dense aggregations of IgG on the SWNT surface (top). Scale bar represents $500 \mathrm{~nm}$. Magnified phase AFM image shows dense aggregates of IgG on the surface of dsSWNT due to non-specific adsorption of IgG on the hydrophobic sidewall of SWNT (bottom). Scale bar represents $100 \mathrm{~nm}$.

protein size could be explained by the drying induced reduction in the overall protein dimension during AFM sample preparations. According to the estimated heights of IgG (i.e., $\sim 3.5 \mathrm{~nm}$ ) and iAmp-SWNTs (i.e., $\sim 1.2 \mathrm{~nm}$ ), it is assumed that the around 6-fold increase in diameter after $\mathrm{Ab}$ conjugation can be attributed to IgG conjugating to SWNTs $(\sim 2+1)$ along its 3D circumference. During the bioconjugation reactions, we again noticed that the iAmp-SWNTs remarkably resisted the nonspecific adsorption of proteins. As seen in Fig. $4 \mathrm{~d}$, when IgG was admixed with iAmp-SWNTs, no association between the IgG molecules and iAmp-SWNT adducts was observed. However, when IgG was admixed with chemically oxidized SWNTs, i.e., dsSWNTs, without iAmp, dense aggregations of IgG on the SWNT surface were noticeable (Fig. 4e). The absence of dense aggregations of protein moieties on the iAmp-SWNT sidewall reconfirms that nonspecific hydrophobic interactions are profoundly minimized through iAmp treatment. We were curious if the iAmp coating could prevent SWNTs against recognition by cells of the MPS, such as macrophages, by preventing nonspecific adsorption of plasma proteins or opsonins.

\section{Macrophage-opsonin model of iAmp-SWNT evasion}

Complement factor, C3b, and immunoglobulin, IgG, are the most abundant opsonins found in human serum with concentrations of $0.55 \mathrm{mg} \mathrm{ml}{ }^{-1}$ and $8 \mathrm{mg} \mathrm{ml}^{-1}$, respectively. ${ }^{45} \mathrm{~A}$ single macrophage possesses 2 million receptors for opsonins. ${ }^{46}$ Therefore, in order to achieve a reasonable balance, the ratio of an opsonin to a macrophage was fixed at $10^{6}: 1$. We assumed that an opsonin/SWNT concentration ratio of $4: 1$ would ensure tagging of all the SWNTs in solution. The IgG was labeled with a fluorescent dye, fluorescein isothiocyanate (FITC), to enable the tracking of the dsSWNT control and iAmp-SWNT complexes in vitro for their interactions with the opsonin. The particles were preincubated with the opsonins, IgG-FITC and/or C3b, before introducing them into macrophages, to ensure their interactions with the opsonins. The samples pre-conjugated with IgG and IgG-FITC were also assessed to confirm the downstream targeting functionality. Using fluorescence microscopy, the localizations of particles with respect to macrophages were evaluated. Macrophages incubated with the control, dsSWNTs, after preincubations with both IgG-FITC and C3b or IgG-FITC alone, remain highly fluorescent (Fig. 5a, right). This is indicative of the opsonization of dsSWNTs and subsequent internalization by the macrophages. Light microscopy revealed a grainy cytoplasm and an irregular cell membrane (Fig. 5a, left), characteristic signs of 'loaded' cells (Fig. 5d, schematic). A similar irregularity was noticed in macrophages with dsSWNTs after preincubations with C3b only (Fig. S11 in the ESI†े). However, there was no fluorescence observed in macrophages incubated with iAmpSWNTs in the presence of opsonins (Fig. 5b, right) and no irregularity was noticed from macrophages (Fig. 5b, left). Also, there was no uptake of trypan blue dye by these cells proving that the cells were viable and the iAmp-SWNT constructs were nontoxic. Trypan blue is a vital stain used to stain dead cells blue. Similarly, there was no significant intracellular fluorescence emanating from macrophages when tested with the IgG-conjugated iAmp-SWNTs, except for the hardly distinct autofluorescence of cells and backgrounds in the emission/ excitation range of the DAPI filter (Fig. S12 in the ESI $\dagger$ ). Furthermore, no fluorescence was observed in macrophages when 
a)
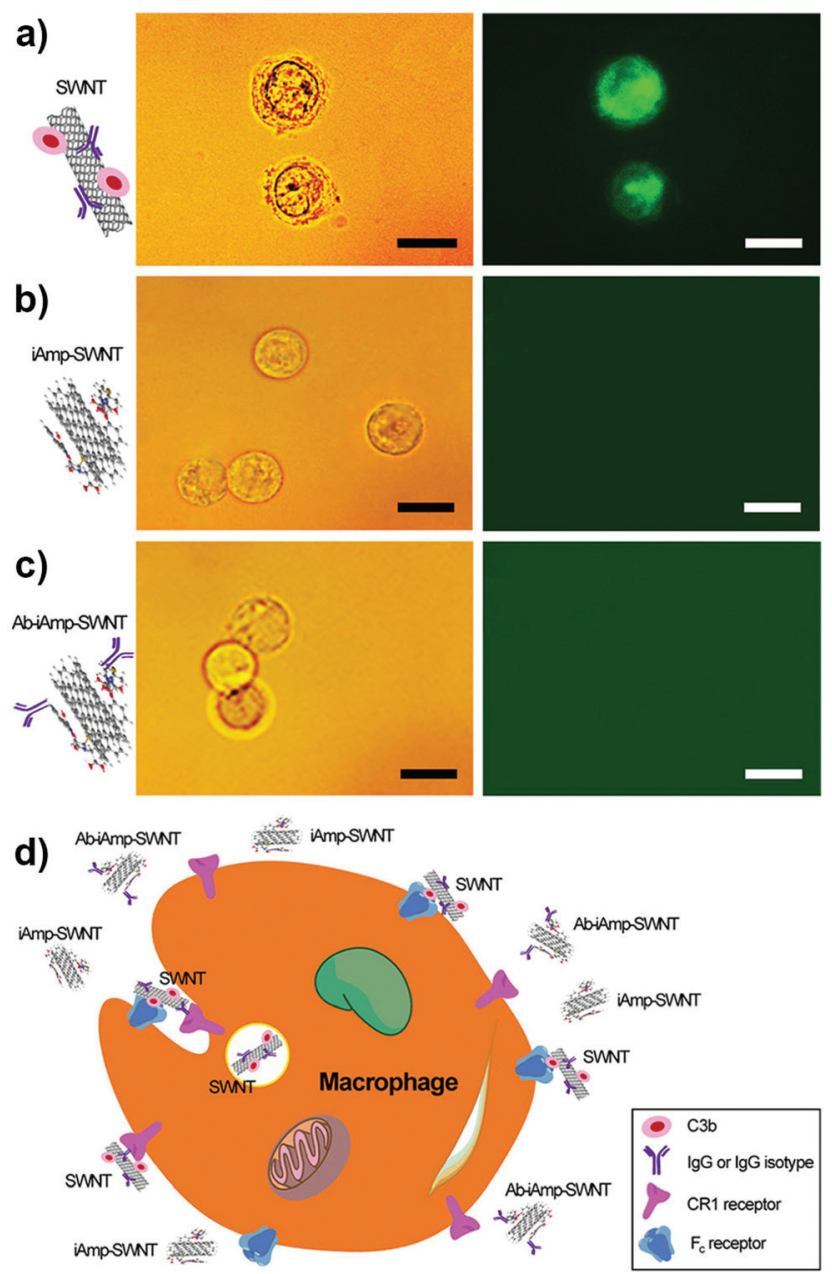

Fig. 5 Light (left) and epi-fluorescence (right) microscopy images of macrophages with: uncoated dsSWNTs (a), iAmp-SWNTs (b), and iAmpSWNT-IgG-FITC (c). Uncoated dsSWNTs and iAmp-SWNT hybrids were preincubated with opsonins, i.e., C3b and/or IgG-FITC. Scale bar represents $25 \mu \mathrm{m}$. (d) Schematic illustrating stealth characters afforded by coating SWNTs with iAmp. Macrophages recognize uncoated dsSWNTs tagged by $\mathrm{C} 3 \mathrm{~b}$ and/or IgG through $\mathrm{CR} 1$ and $\mathrm{F}_{\mathrm{c}}$ receptors, respectively. Subsequently, macrophages phagocytize the opsonized SWNTs. However, iAmp is shielding the SWNTs from opsonins, preventing phagocytosis by macrophages. Also, the majority of the conjugated IgG to iAmp-SWNTs are oriented with the $\mathrm{F}_{\mathrm{c}}$ region concealed, alleviating recognition by macrophages. Note that the schematics do not reflect the actual object sizes.

assessed with iAmp-SWNT-IgG-FITC to avoid any possibility of masking and attenuation of iAmp fluorescence by the background autofluorescence (Fig. 5c). These results demonstrate that iAmp is shielding the SWNTs from opsonins and eventually preventing phagocytosis by macrophages (Fig. $5 \mathrm{~d}$ ). This phenomenon can be mainly attributed to the decrease in van der Waals and hydrophobic forces on the SWNT surface, which are primarily implicated in their strong interaction with cell surfaces. While PEGylation is also known to efficiently shield SWNTs from opsonins, by itself it is not an efficient dispersant and does not fluoresce, therefore lacking the versatility of iAmp.
As reported in our earlier work, ${ }^{24}$ we hypothesized that either IgG or C3b could be implicated in exclusively tagging foreign particles and results in particles being recognized and phagocytized by macrophages. Based on the results in this study, we suggest the following. (1) Particles tagged by C3b or IgG alone can be primed for recognition and internalization by macrophages. Particularly, since complement receptor 1 (CR1), on the surface of macrophages, does not require C3b to be in any particular orientation for recognition and binding purposes, it is important that SWNTs and nanoparticles in general have an opsonin resistant coating. (2) The reason the Ab-conjugated iAmp-SWNT was not internalized even though it was functionalized with IgG, has to do with the orientation of the IgG on the SWNT surface. Macrophages have receptors for the $F_{c}$ region of IgG. No internalization will occur if the orientation of $\mathrm{Ab}$ on the SWNT is such that the $\mathrm{F}_{\mathrm{c}}$ region is inaccessible for binding to the receptors. There is no such guarantee for the nonspecifically adsorbed $\mathrm{Ab}$ as noticed with the dsSWNT control in the presence of IgG only, suggesting that careful consideration should be given while designing probe and targeting systems that use $\mathrm{Ab}$ as a homing moiety. Our site-specific $\mathrm{Ab}$ conjugation strategy on the surface of iAmp-SWNTs did not lead to internalization by macrophages. We can therefore infer that the majority of the conjugated IgG were oriented with the $\mathrm{F}_{\mathrm{c}}$ region concealed or not conducive for recognition by macrophages. Also, our Ab functionalization, using tailored surface chemistry through a specific covalent linkage, enables the $\mathrm{F}_{\mathrm{ab}}$ region to be favorably oriented and to retain its binding capacity for molecularly targeted theranostics.

\section{Conclusion}

In this study, we have identified a multifunctional fluorescent derivative of the beta-lactam antibiotic ampicillin, i.e., iAmp, which has significant implications for improving the in vivo effectiveness of theranostic materials. It was easy to synthesize, through a one-step procedure, and to process for coating nanoparticles, such as SWNTs. It imparted several of its unique traits such as solubility, bio-stability, fluorescence, $\mathrm{pH}$ sensitivity, inert structure, and biocompatibility to SWNTs, which are very important for translation into biological and biomedical practices. The high fluorescence quantum yield along with its stability against precipitation and photobleaching under ambient conditions could qualify the iAmp as a fluorophore for several applications. Its fluorescence was quenched in an acidic environment but recovered completely under neutral and basic conditions. The antibacterial properties of its parent compound were attenuated and viability tests showed that iAmp has minimal toxicity. The structure resembled that of ampicillin except for the amine group at $\mathrm{H}-10$ replaced by a carbonyl group. The iAmp improved the solubility and assisted the uniform dispersion of SWNTs as shown through characteristic Van Hove peaks in absorbance readings and AFM analyses. Its coating through $\pi-\pi$ interaction 
drastically improved the biocompatibility of SWNTs with minimal cytotoxicity, remained stable under biologically relevant conditions with high protein and salt concentrations, and rendered SWNTs notable resistances against nonspecific adsorptions of proteins and cells. The iAmp-SWNT adducts were visualized through epi-fluorescence microscopy, which makes it feasible to track SWNTs in situ and perform real time diffusion based studies in physiological solutions. A sitespecific bioconjugation of Ab could be achieved through tailored surface chemistry, facilitating highly specific target binding. The macrophage-opsonin model revealed that the iAmp coating afforded stealth characters to SWNTs and effectively prevented nonspecific adsorption of opsonins, IgG and/ or $\mathrm{C} 3 \mathrm{~b}$, and phagocytosis by macrophages. This suggests a potential long circulation time of iAmp-SWNTs in vivo with transparency against nonspecific protein and cell adsorptions. Hence, although further validation is required in in vivo animal models, which are currently underway, iAmp has shown high promise to facilitate cancer nanotheranostics as it would not only improve the circulation half-life and biodistribution of nanomaterials but also impart useful traits to the material for treating and imaging cancer.

\section{Acknowledgements}

This work was supported in part by the National Science Foundation (CMMI 1235100, ECCS 1128660 and OIA 1457888), the National Institutes of Health (GM103429 and GM103450), and the Arkansas Biosciences Institute. The authors acknowledge discussions and technical assistance from Drs Jackson O. Lay and Ju Seok Lee, and thank Ryan K.-H. Kim for his assistance in image processing.

\section{Notes and references}

1 C. A. Schütz, L. Juillerat-Jeanneret, H. Mueller, I. Lynch and M. Riediker, Nanomedicine, 2013, 8, 449-467.

2 E.-K. Lim, T. Kim, S. Paik, S. Haam, Y.-M. Huh and K. Lee, Chem. Rev., 2015, 115, 327-394.

3 N. Kotagiri and J.-W. Kim, Int. J. Nanomed., 2014, 1, 85-105.

4 D. Liu, Q. Hu and Y. K. Song, Biochim. Biophys. Acta, Biomembr., 1995, 1240, 277-284.

5 M. P. Monopoli, C. Aberg, A. Salvati and K. A. Dawson, Nat. Nanotechnol., 2012, 7, 779-786.

6 S. Dumitriu, Polymeric Biomaterials, Revised and Expanded, CRC Press, 2001.

7 S. Stolnik, L. Illum and S. S. Davis, Adv. Drug Delivery Rev., 1995, 16, 195-214.

8 R. H. Baughman, A. A. Zakhidov and W. A. de Heer, Science, 2002, 297, 787-792.

9 V. P. Zharov, J.-W. Kim, D. T. Curiel and M. Everts, Nanomedicine, 2005, 1, 326-345.

10 N. W. Kam, M. O’Connell, J. A. Wisdom and H. Dai, Proc. Natl. Acad. Sci. U. S. A., 2005, 102, 11600-11605.
11 J.-W. Kim, E. V. Shashkov, E. I. Galanzha, N. Kotagiri and V. P. Zharov, Lasers Surg. Med., 2007, 39, 622-634.

12 V. P. Zharov, E. I. Galanzha, E. V. Shashkov, J.-W. Kim, N. G. Khlebtsov and V. V. Tuchin, J. Biomed. Opt., 2007, 12, 051503.

13 L. De, C. Zavaleta, S. Keren, S. Vaithilingam, S. Bodapati, Z. Liu, J. Levi, B. Smith, T.-J. Ma, O. Oralkan, Z. Cheng, X. Chen, H. Dai, B. Khuri-Yakub and S. Gambhir, Nat. Nanotechnol., 2008, 3, 557-562.

14 J.-W. Kim, E. I. Galanzha, E. V. Shashkov, H. M. Moon and V. P. Zharov, Nat. Nanotechnol., 2009, 4, 688-694.

15 E. I. Galanzha, E. V. Shashkov, T. Kelly, J.-W. Kim, L. Yang and V. P. Zharov, Nat. Nanotechnol., 2009, 4, 855860.

16 E. I. Galanzha, M. S. Kokoska, E. V. Shashkov, J.-W. Kim, V. V. Tuchin and V. P. Zharov, J. Biophotonics, 2009, 2, 528539.

17 E. I. Galanzha, J.-W. Kim and V. P. Zharov, J. Biophotonics, 2009, 2, 725-735.

18 N. Kotagiri and J.-W. Kim, Macromol. Biosci., 2010, 10, 231238.

19 J.-W. Kim, H. M. Moon, M. Benamara, J. Sakon, G. J. Salamo and V. P. Zharov, Chem. Commun., 2010, 46, 7142-7144.

20 A. de la Zerda, J.-W. Kim, E. I. Galanzha, S. S. Gambhir and V. P. Zharov, Contrast Media Mol. Imaging, 2011, 6, 346369.

21 E. I. Galanzha, E. Shashkov, M. Sarimollaoglu, K. E. Beenken, A. G. Basnakian, M. E. Shirtliff, J.-W. Kim, M. S. Smeltzer and V. P. Zharov, PLoS One, 2012, 7, e45557.

22 J.-W. Kim, E. I. Galanzha, D. A. Zaharoff, R. J. Griffin and V. P. Zharov, Mol. Pharm., 2013, 10, 813-830.

23 J.-W. Kim and R. Deaton, Part. Part. Syst. Charact., 2013, 30, 117-132.

24 N. Kotagiri, J. S. Lee and J.-W. Kim, J. Biomed. Nanotechnol., 2013, 9, 1008-1016.

25 C. A. Poland, R. Duffin, I. Kinloch, A. Maynard, W. A. Wallace, A. Seaton, V. Stone, S. Brown, W. Macnee and K. Donaldson, Nat. Nanotechnol., 2008, 3, 423428.

26 R. Singh, D. Pantarotto, L. Lacerda, G. Pastorin, C. Klumpp, M. Prato, A. Bianco and K. Kostarelos, Proc. Natl. Acad. Sci. U. S. A., 2006, 103, 3357-3362.

27 P. Wick, P. Manser, L. K. Limbach, U. Dettlaff-Weglikowska, F. Krumeich, S. Roth, W. J. Stark and A. Bruinink, Toxicol. Lett., 2007, 168, 121-131.

28 M. Zheng, A. Jagota, E. D. Semke, B. A. Diner, R. S. McLean, S. R. Lustig, R. E. Richardson and N. G. Tassi, Nat. Mater., 2003, 2, 338-342.

29 F. Balavoine, P. Schultz, C. Richard, V. Mallouh, T. W. Ebbesen and C. Mioskowski, Angew. Chem., Int. Ed., 1999, 38, 1912-1915.

30 J.-W. Kim, N. Kotagiri, J.-H. Kim and R. Deaton, Appl. Phys. Lett., 2006, 88, 213110.

31 A. J. Blanch, C. E. Lenehan and J. S. Quinton, J. Phys. Chem. $B, 2010$, 114, 9805-9811. 
32 P. Cherukuri, C. J. Gannon, T. K. Leeuw, H. K. Schmidt, R. E. Smalley, S. A. Curley and R. B. Weisman, Proc. Natl. Acad. Sci. U. S. A., 2006, 103, 18882-18886.

33 Y. Zhang, Y. Xu, Z. Li, T. Chen, S. M. Lantz, P. C. Howard, M. G. Paule, W. Slikker Jr., F. Watanabe, T. Mustafa, A. S. Biris and S. F. Ali, ACS Nano, 2011, 5, 70207033.

34 S. M. Moghimi, A. J. Andersen, S. H. Hashemi, B. Lettiero, D. Ahmadvand, A. C. Hunter, T. L. Andresen, I. Hamad and J. Szebeni, J. Controlled Release, 2010, 146, 175-181.

35 T. Uno, M. Masada, Y. Kuroda and T. Nakagawa, Chem. Pharm. Bull., 1981, 29, 1344-1354.

36 S. A. Carabineiro, T. Thavorn-Amornsri, M. F. Pereira and J. L. Figueiredo, Water Res., 2011, 45, 4583-4591.

37 A. S. Kumar, S. Sornambikai, L. Deepika and J.-M. Zen, J. Mater. Chem., 2010, 20, 10152-10158.

38 L. Ji, W. Chen, S. Zheng, Z. Xu and D. Zhu, Langmuir, 2009, 25, 11608-11613.
39 Z. Liu, A. C. Fan, K. Rakhra, S. Sherlock, A. Goodwin, X. Chen, Q. Yang, D. W. Felsher and H. Dai, Angew. Chem., Int. Ed., 2009, 48, 7668-7672.

40 J. Judkins, H. H. Lee, S. Tung and J.-W. Kim, J. Biomed. Nanotechnol., 2013, 9, 1065-1070.

41 F. W. McLafferty and F. Tureček, Interpretation of Mass Spectra, University Science Books, 1993.

42 R. J. Chen, Y. Zhang, D. Wang and H. Dai, J. Am. Chem. Soc., 2001, 123, 3838-3839.

43 L. Liu, T. Wang, J. Li, Z.-X. Guo, L. Dai, D. Zhang and D. Zhu, Chem. Phys. Lett., 2003, 367, 747-752.

44 Y. H. Tan, M. Liu, B. Nolting, J. G. Go, J. Gervay-Hague and G. Y. Liu, ACS Nano, 2008, 2, 2374-2384.

45 M. M. Wintrobe and J. P. Greer, Wintrobe's Clinical Hematology, Wolters Kluwer Health/Lippincott Williams \& Wilkins, 2009.

46 W. P. Arend and M. Mannik, J. Immunol., 1973, 110, 14551463. 